\title{
UNIQUENESS OF NON-TOPOLOGICAL SOLUTIONS FOR THE CHERN-SIMONS SYSTEM WITH TWO HIGGS PARTICLES
}

\author{
Hsin-Yuan Huang and Chang-Shou Lin
}

\begin{abstract}
We study the non-topological radial solutions of the Abelian Chern-Simons equation with two Higgs particles. We establish the non-degeneracy property of linearized equation and the uniqueness property for the corresponding non-topological radial solutions.
\end{abstract}

\section{Introduction}

The Chern-Simons theories were developed to explain certain particle physics, condensed physics, superconductivity, quantum mechanics and so on. The Chern-Simons equations of various models correspond to non-linear elliptic equations, which are both interesting and challenging.

In this paper, we consider the non-linear elliptic system

$$
\left\{\begin{array}{l}
\Delta u+e^{v}\left(1-e^{u}\right)=\mu \\
\Delta v+e^{u}\left(1-e^{v}\right)=v
\end{array} \text { in } \mathbf{R}^{2}\right.
$$

where $\mu$ and $v$ are finite measure of the form $4 \pi \sum_{s} \delta_{p_{s}}$ on $\mathbf{R}^{2}$. This system arises in a relativistic Abelian Chern-Simons model involving two Higgs scalar fields and two gauge fields. We refer to [8] for the details on the derivation of this system and $[6,7]$ for the background physics. For the past twenty years, the Abelian Chern-Simons equation with one Higgs particle,

$$
\Delta u+e^{u}\left(1-e^{u}\right)=4 \pi \sum_{s} \delta_{p_{s}}
$$

has been extensively studied. We refer reader to $[13,1,14,2,12,3,5,10]$ and references therein for the recent developments.

In the literature, there are two natural boundary conditions for the solutions of (1.1) at infinity, namely,

(1) $\lim _{|x| \rightarrow \infty} u(x)=\lim _{|x| \rightarrow \infty} v(x)=0$

(2) $\lim _{|x| \rightarrow \infty} u(x)=\lim _{|x| \rightarrow \infty} v(x)=-\infty$

2010 Mathematics Subject Classification. 35J60, 35 J57.

Key words and phrases. Chern-Simons System, Uniqueness, Non-Topological Solutions.

Received May 23, 2013. 
If $(u, v)$ satisfies the boundary condition $(1)$, then $(u, v)$ is called a topological solution of (1.1).

In [8], Lin-Ponce-Yang showed the existence of topological solutions for (1.1) for any given set of singularities.

THEOREM 1.1 ([8]). For any given sets $\left\{p_{1}, \ldots, p_{N_{1}}\right\}$ and $\left\{q_{1}, \ldots, q_{N_{2}}\right\}$ on $\mathbf{R}^{2}$, (1.1) with $\mu=4 \pi \sum_{i=1}^{N_{1}} \delta_{p_{i}}$ and $v=4 \pi \sum_{j=1}^{N_{2}} \delta_{q_{j}}$ possesses a topological solution.

Lin-Prajapat [9] proved the existence of maximal and mountain-pass solutions of (1.1) on a torus. Lin-Yan [11] studied the bubbling solutions of (1.1) on a torus. Chern-Chen-Lin [4] studied the radial solutions of (1.1) with all $\left\{p_{i}\right\}_{i=1}^{N_{1}}$ and $\left\{q_{j}\right\}_{j=1}^{N_{2}}$ to be the origin.

$$
\left\{\begin{array}{l}
\Delta u+e^{v}\left(1-e^{u}\right)=4 \pi N_{1} \delta_{0} \\
\Delta v+e^{u}\left(1-e^{v}\right)=4 \pi N_{2} \delta_{0}
\end{array} \text { in } \mathbf{R}^{2} .\right.
$$

They showed the uniqueness of topological solutions of (1.2) by studying the nondegeneracy property of linearized equations and classify all entire radial solutions of (1.2) according to their behaviours at $\infty$ :

Type (I): $\lim _{r \rightarrow \infty}(u(r), v(r))=(0,0)$.

Type (II): $\lim _{r \rightarrow \infty}(u(r), v(r))=(-\infty,-\infty)$ with $\beta_{1}<\infty$ and $\beta_{2}<\infty$. For this case, $(u, v)$ is called a non-topological solution.

Type (III): $\lim _{r \rightarrow \infty}(u(r), v(r))=(-\infty,-\infty)$, and either $2 N_{1}<\beta_{1} \leq 2 N_{1}+2$, $\beta_{2}=\infty$ or $\beta_{1}=\infty, 2 N_{2}<\beta_{2} \leq 2 N_{2}+2$.

Type (IV): $\lim _{r \rightarrow \infty}(u(r), v(r))=\left(-c_{u}, \infty\right)$ or $\lim _{r \rightarrow \infty}(u(r), v(r))=\left(-\infty,-c_{v}\right)$ for some constants $c_{u}>0$ and $c_{v}>0$. Here,

Type (V): $\lim _{r \rightarrow \infty}(u(r), v(r))=(+\infty,-\infty)$ or $\lim _{r \rightarrow \infty}(u(r), v(r))=(-\infty,+\infty)$.

$$
\beta_{1}=\int_{0}^{\infty} e^{v}\left(1-e^{u}\right) r d r, \quad \beta_{2}=\int_{0}^{\infty} e^{u}\left(1-e^{v}\right) r d r
$$

However, the issues of the uniqueness of the non-topological solutions have not been understood yet.

In this paper, we consider the radial solution of (1.1) with $\mu=v=0$ :

$$
\left\{\begin{array}{l}
\Delta u+e^{v}\left(1-e^{u}\right)=0 \\
\Delta v+e^{u}\left(1-e^{v}\right)=0
\end{array} \text { in } \mathbf{R}^{2} .\right.
$$

The following theorem is the main theorem of this paper. We prove the uniqueness result for the non-topological radial solutions of (1.3).

THEOREM 1.2. For any given pair $\left(\beta_{1}, \beta_{2}\right)$ with $2<\beta_{i}<\infty, i=1,2$, and $\left(\beta_{1}-2\right)\left(\beta_{2}-2\right)>4$, there exists a unique non-topological radial solution of $(1.3)$ satisfies

$$
\int_{0}^{\infty} e^{v}\left(1-e^{u}\right) r d r=\beta_{1} \quad \text { and } \quad \int_{0}^{\infty} e^{u}\left(1-e^{v}\right) r d r=\beta_{2} .
$$


The proof is based on the non-degeneracy of linearized equations. The linearized equations at $(u, v)$ of $(1.2)$ is called degenerate if there exists a nonzero bounded solution $(A, B)$ of

$$
\left\{\begin{array}{l}
\Delta A+e^{v}\left(1-e^{u}\right) B-e^{u+v} A=0 \\
\Delta B+e^{u}\left(1-e^{v}\right) A-e^{u+v} B=0
\end{array} \text { in } \mathbf{R}^{2} .\right.
$$

In Sec. 2, we will show the non-degeneracy of linearized equations at a nontopological solution. We hope that the method developed here could be helpful for a similar non-linear elliptic systems, like $A_{2}, B_{2}$ and $G_{2}$ Chern-Simons system. For the single Chern-Simons-Higgs model, the uniqueness result for the nontopological solution with one singularity at the origin was proved in [3]. Is there any uniqueness result for the non-topological solutions of (1.2) with $\left(\beta_{1}, \beta_{2}\right)$ satisfying

$$
\left(\beta_{1}-2\left(N_{1}+1\right)\right)\left(\beta_{2}-2\left(N_{2}+1\right)\right)>4\left(N_{1}+1\right)\left(N_{2}+1\right) ?
$$

We will come back to this issue in a coming paper.

The paper is organized as follows. We investigate the non-degeneracy property of the linearized equations on the non-topological solutions of (1.3) in Sec. 2. Theorem 1.2 is proved in Sec. 3.

\section{Non-degeneracy of linearized equations}

In this section, we consider the radial solutions of

$$
\left\{\begin{array}{l}
\Delta u+e^{v}\left(1-e^{u}\right)=0 \\
\Delta v+e^{u}\left(1-e^{v}\right)=0
\end{array} \text { in } \mathbf{R}^{2} .\right.
$$

Denote $\left(u\left(r ; \alpha_{1}, \alpha_{2}\right), v\left(r ; \alpha_{1}, \alpha_{2}\right)\right.$ be the solution of $(2.1)$ with $(u(0), v(0))=\left(\alpha_{1}, \alpha_{2}\right)$ and

$$
\begin{aligned}
& \beta_{1}\left(\alpha_{1}, \alpha_{2}\right)=\int_{0}^{\infty} e^{v\left(r ; \alpha_{1}, \alpha_{2}\right)}\left(1-e^{u\left(r ; \alpha_{1}, \alpha_{2}\right)}\right) r d r \\
& \beta_{2}\left(\alpha_{1}, \alpha_{2}\right)=\int_{0}^{\infty} e^{u\left(r ; \alpha_{1}, \alpha_{2}\right)}\left(1-e^{v\left(r ; \alpha_{1}, \alpha_{2}\right)}\right) r d r
\end{aligned}
$$

Remark 2.1. For any solution of (2.1) with $\beta_{1}<+\infty$ and $\beta_{2}<+\infty$, we can prove that $(u, v)$ is symmetric with respect to some point $p \in \mathbf{R}^{2}$. The proof can be obtained via the method of moving planes.

Denote the set of the initial conditions of the non-topological solutions of (2.1).

$$
\begin{aligned}
\Omega= & \left\{\left(\alpha_{1}, \alpha_{2}\right) \in \mathbf{R}^{2} \mid u(0)=\alpha_{1}, v(0)=\alpha_{2},\right. \\
& \text { such that } \left.u(r)<0 \text { and } v(r)<0 \text { and } \beta_{1}<+\infty, \beta_{2}<+\infty\right\}
\end{aligned}
$$

In [4], Chern-Chen-Lin prove 
Proposition 2.2. $\Omega$ is simply connected.

Let

$$
\begin{aligned}
& \phi_{i}(r)=\frac{\partial u}{\partial \alpha_{i}}, \quad i=1,2 . \\
& \psi_{i}(r)=\frac{\partial v}{\partial \alpha_{i}}, \quad i=1,2 .
\end{aligned}
$$

Then $\left(\phi_{i}, \psi_{i}\right), i=1,2$ satisfied the linearized equation

$$
\left\{\begin{array}{l}
\Delta \phi_{i}-e^{u+v} \phi_{i}+e^{v}\left(1-e^{u}\right) \psi_{i}=0 \\
\Delta \psi_{i}-e^{u+v} \psi_{i}+e^{u}\left(1-e^{v}\right) \phi_{i}=0 \\
\phi_{1}(0)=\psi_{2}(0)=1, \phi_{2}(0)=\psi_{1}(0)=0, \phi_{i}^{\prime}(0)=\psi_{i}^{\prime}(0)=0 .
\end{array}\right.
$$

By [4], we have the monotone property of $\left(\phi_{i}, \psi_{i}\right), i=1,2$.

Proposition 2.3. $\phi_{1}(r)>0$ and $\psi_{2}(r)>0$ for $r \geq 0$, and $\phi_{2}(r)<0$ and $\psi_{1}(r)<0$ for $r \geq 0$ provided that $\left(\alpha_{1}, \alpha_{2}\right) \in \Omega$.

It is not difficult to show that

$$
\begin{aligned}
& \phi_{1}(r)=A_{1} \log r+O(1), \quad \phi_{2}(r)=-B_{1} \log r+O(1), \\
& \psi_{1}(r)=-B_{2} \log r+O(1), \quad \psi_{2}(r)=A_{2} \log r+O(1),
\end{aligned}
$$

as $r \rightarrow+\infty$, for some $A_{i}>0$ and $B_{i}>0, i=1,2$. More precisely,

$$
\begin{aligned}
& A_{1}=\int_{0}^{\infty}\left(e^{u+v} \phi_{1}-e^{v}\left(1-e^{u}\right) \psi_{1}\right) r d r, \quad B_{1}=\int_{0}^{\infty}\left(-e^{u+v} \phi_{2}+e^{v}\left(1-e^{u}\right) \psi_{2}\right) r d r, \\
& B_{2}=\int_{0}^{\infty}\left(-e^{u+v} \psi_{1}+e^{u}\left(1-e^{v}\right) \phi_{1}\right) r d r, \quad A_{2}=\int_{0}^{\infty}\left(e^{u+v} \psi_{2}-e^{u}\left(1-e^{v}\right) \phi_{2}\right) r d r .
\end{aligned}
$$

It is clear that both $\left(\phi_{1}, \psi_{1}\right)$ and $\left(\phi_{2}, \psi_{2}\right)$ satisfy the linearized equation (1.4). In fact, any solution $(\phi, \psi)$ of the linearized equation comes from a linear combination of $\left(\phi_{1}, \psi_{1}\right)$ and $\left(\phi_{2}, \psi_{2}\right)$.

We want to show that the linearized equation is non-degenerate. It is not difficult to see it is equivalent to saying that for any solution $(\phi, \psi)$ of the linearized equation, $|\phi(x)|+|\psi(x)|$ is not bounded in $[0, \infty)$. Thus, it is equivalent to

$$
\operatorname{det}\left(\begin{array}{cc}
A_{1} & -B_{1} \\
-B_{2} & A_{2}
\end{array}\right) \neq 0
$$

We will utilize the following the Pohozaev's identity for (1.3) to prove it.

$$
\int_{0}^{R}\left(e^{v}+e^{u}-e^{u+v}\right) r d r=\frac{R^{2} u^{\prime}(R) v^{\prime}(R)+R^{2}\left(e^{u(R)}+e^{v(R)}-e^{(u+v)(R)}\right)}{2} .
$$


By differentiating the above identity with respect to the initial value of $(u, v)$, we have

$$
\begin{aligned}
\int_{0}^{R}\left(e^{u} \phi\right. & \left.+e^{v} \psi-e^{u} e^{v}(\phi+\psi)\right) r d r \\
= & \frac{R^{2}}{2}\left[u^{\prime}(R) \psi^{\prime}(R)+v^{\prime}(R) \psi^{\prime}(R)\right] \\
& +\frac{R^{2}}{2}\left[e^{u(R)} \phi(R)+e^{v(R)} \psi(R)-e^{(u+v)(R)}(\psi+\psi)(R)\right] .
\end{aligned}
$$

TheOREM 2.4 .

$$
\operatorname{det}\left(\begin{array}{cc}
A_{1} & -B_{1} \\
-B_{2} & A_{2}
\end{array}\right) \neq 0 .
$$

Proof. Without loss of generality, we assume that

$$
(u, v)(0)=\left(\alpha_{1}^{*}, \alpha_{2}^{*}\right) \text { with } \alpha_{2}^{*}>\alpha_{1}^{*} .
$$

In the followings, we set

$$
\phi_{c}\left(r ; \alpha_{1}, \alpha_{2}\right)=\phi_{1}\left(r ; \alpha_{1}, \alpha_{2}\right)+c \phi_{2}\left(r ; \alpha_{1}, \alpha_{2}\right)
$$

and

$$
\psi_{2}\left(r ; \alpha_{1}, \alpha_{2}\right)=\psi_{1}\left(r ; \alpha_{1}, \alpha_{2}\right)+c \psi_{2}\left(r ; \alpha_{1}, \alpha_{2}\right)
$$

for some constant $c$. Then $\left(\phi_{c}, \psi_{c}\right)$ is a solution of the linearized equation. If $c \leq 0$, then it is clear that $\left(\phi_{c}, \psi_{c}\right)$ is unbounded in $[0, \infty)$. Hence, we may only consider the case $c>0$. Let

$$
\begin{array}{r}
\widehat{\alpha_{2}}=\sup \left\{\alpha<\alpha_{2}^{*} \mid \text { For all } \beta \in\left(\alpha_{1}^{*}, \alpha\right], \text { there is } c(\beta)>0\right. \text { such that both } \\
\left.\phi_{c}\left(r ; \alpha_{1}^{*}, \beta\right) \text { and } \psi_{c}\left(r ; \alpha_{1}^{*}, \beta\right) \text { change sign once and only once. }\right\}
\end{array}
$$

STEP 1. If both $\phi_{c}(r)$ and $\psi_{c}(r)$ change sign once and only once, then at least one of them is unbounded on $[0, \infty)$. satisfies

Note that $\phi_{c}(0)=\phi_{1}(0)>0$ and $\psi_{c}(0)=c \psi_{2}(0)>0$. We know that $\left(\phi_{c}, \psi_{c}\right)$

$$
\left\{\begin{array}{l}
\Delta \phi_{c}+e^{v}\left(1-e^{u}\right) \psi_{c}-e^{u+v} \phi_{c}=0 \\
\Delta \psi_{c}+e^{u}\left(1-e^{v}\right) \phi_{c}-e^{u+v} \psi_{c}=0
\end{array}\right.
$$

Now, suppose both $\phi_{c}$ and $\psi_{c}$ are bounded. Then

$$
\int_{0}^{\infty} e^{v}\left(1-e^{u}\right) \psi_{c} r d r=\int_{0}^{\infty} e^{u+v} \phi_{c} r d r
$$


and

$$
\int_{0}^{\infty} e^{u}\left(1-e^{v}\right) \phi_{c} r d r=\int_{0}^{\infty} e^{u+v} \psi_{c} r d r .
$$

By applying the Pohozaev's identity, we have

$$
\int_{0}^{\infty}\left(e^{u} \phi_{c}+e^{v} \psi_{c}\right) r d r=\int_{0}^{\infty} e^{u+v}\left(\phi_{c}+\psi_{c}\right) r d r .
$$

On the other hand, by (2.5) and (2.6),

$$
\int_{0}^{\infty}\left(e^{u} \phi_{c}+e^{v} \psi_{c}\right) r d r=2 \int_{0}^{\infty} e^{u+v}\left(\phi_{c}+\psi_{c}\right) r d r
$$

together with (2.7), it implies

$$
\int_{0}^{\infty} e^{u+v}\left(\phi_{c}+\psi_{c}\right) r d r=0
$$

Again, by (2.5) and (2.6), one obtains

$$
\int_{0}^{\infty} e^{u} \phi_{c} r d r=\int_{0}^{\infty} e^{v} \psi_{c} r d r=0 .
$$

Suppose that $\phi_{c}\left(r_{1}\right)=0$ and $\psi_{c}\left(r_{2}\right)=0$ for some $r_{1}>0$ and $r_{2}>0$. Since $u^{\prime}(r)<0$ and $v^{\prime}(r)<0$ for $r>0$, we have

$$
\left(e^{v\left(r_{1}\right)}-e^{v(r)}\right) \phi_{c}(r)<0 \quad \text { if } r \neq r_{1} .
$$

Hence,

$$
\int_{0}^{\infty} e^{u(r)}\left(e^{v\left(r_{1}\right)}-e^{v(r)}\right) \phi_{c}(r) r d r<0,
$$

and it implies

$$
0=e^{v\left(r_{1}\right)} \int_{0}^{\infty} e^{u(r)} \phi_{c}(r) r d r<\int_{0}^{\infty} e^{(u+v)(r)} \phi_{c}(r) r d r .
$$

Similarly, we have

$$
0=e^{u\left(r_{2}\right)} \int_{0}^{\infty} e^{v(r)} \psi_{c}(r) r d r<\int_{0}^{\infty} e^{(u+v)(r)} \psi_{c}(r) r d r
$$

But it yields

$$
0<\int_{0}^{\infty} e^{(u+v)(r)}\left(\phi_{c}(r)+\psi_{c}(r)\right) r d r=0,
$$

a contradiction. This finishes the step 1 . 
STEP 2. Suppose $\phi_{c}(r)$ and $\psi_{c}(r)$ change sign only once. Then $\phi_{c}(r)=0$ and $\psi_{c}(r)=0$ has only one solution.

We want to prove if $\phi_{c}\left(r_{0}\right)=0$, then $\phi_{c}^{\prime}\left(r_{0}\right)<0$. Suppose not, i.e. $\phi_{c}^{\prime}\left(r_{0}\right)=0$. Then there is $r_{1}>r_{0}$ such that $\phi_{c}\left(r_{1}\right)>0$ and $r_{1}$ is a local maximum point of $\phi_{c}$.

Since

$$
0 \leq \Delta \phi_{c}\left(r_{0}\right)=e^{v\left(r_{0}\right)}\left(e^{u\left(r_{0}\right)}-1\right) \psi_{c}\left(r_{0}\right),
$$

one has

$$
\psi_{c}\left(r_{0}\right) \leq 0
$$

If $\psi_{c}\left(r_{0}\right)=0$, then we have $\psi_{c}^{\prime}\left(r_{0}\right) \neq 0$. Otherwise, $\left(\phi_{c}, \psi_{c}\right)$ satisfies a second order system of equations with $\phi_{c}\left(r_{0}\right)=\phi_{c}^{\prime}\left(r_{0}\right)=0$ and $\psi_{c}\left(r_{0}\right)=\psi_{c}^{\prime}\left(r_{0}\right)=0$. By the uniqueness of ODE, we have $\phi_{c} \equiv \psi_{c} \equiv 0$, a contradiction. Thus, $\psi_{c}^{\prime}\left(r_{0}\right)<0$.

Hence, we conclude that either $\psi_{c}\left(r_{0}\right)=0$ and $\psi_{c}^{\prime}\left(r_{0}\right)<0$, or $\psi_{c}\left(r_{0}\right)<0$. It is easy to see that either case implies $\psi_{c}\left(r_{1}\right) \leq 0$, due to the fact that $\psi_{c}$ changes sign only once.

By the maximum principle,

$$
0<-\Delta \phi_{c}\left(r_{1}\right)+e^{(u+v)\left(r_{1}\right)} \phi_{c}\left(r_{1}\right)=e^{v\left(r_{1}\right)}\left(1-e^{u\left(r_{1}\right)}\right) \psi_{c}\left(r_{1}\right) \leq 0,
$$

because $\psi_{c}\left(r_{1}\right)<0$ and $u\left(r_{1}\right)<0$. This yields a contradiction and then the step 2 is proved.

STEP 3. $\alpha_{2}^{*}=\widehat{\alpha_{2}}$.

We denote $S=\left\{\beta \mid\right.$ Both $\phi_{c}\left(r ; \alpha_{1}^{*}, \beta\right)$ and $\psi_{c}\left(r ; \alpha_{1}^{*}, \beta\right)$ change sign once and only once for some $c>0\}$. Clearly, $\widehat{\alpha_{2}} \leq \alpha_{2}^{*}$. If $\widehat{\alpha_{2}}<\alpha_{2}^{*}$, then by definition, there exists $c_{i}=c\left(\beta_{i}\right)>0$ such that $\left(\phi_{c_{i}}, \psi_{c_{i}}\right)=\left(\phi_{c_{i}}\left(r ; \alpha_{1}^{*}, \beta_{i}\right), \psi_{c_{i}}\left(r ; \alpha_{1}^{*}, \beta_{i}\right)\right)$ changes sign once and only once, where $\left(\phi_{c_{i}}(r), \psi_{c_{i}}(r)\right)$ is a solution of the linearized equation, where $\beta_{i} \rightarrow \widehat{\alpha_{2}}$. It is easy to see that $\varepsilon \leq c_{i} \leq \varepsilon^{-1}$ for some constant $\varepsilon>0$. By passing to the limit, we find that $\left(\phi_{c}, \psi_{c}\right)$ is a solution of the linearized equation at $(u, v)=\left(u\left(r, \alpha_{1}, \widehat{\alpha_{2}}\right), v\left(r, \alpha_{1}, \widehat{\alpha_{2}}\right)\right)$, and $\phi_{c}$ and $\psi_{c}$ change their sign at most once. We want to prove both $\phi_{c}$ and $\psi_{c}$ cannot be bounded. If both $\phi_{c}$ and $\psi_{c}$ are bounded, then we have

$$
\int_{0}^{\infty} e^{u} \phi_{c}(r) r d r=\int_{0}^{\infty} e^{v} \psi_{c}(r) d r=0
$$

which implies both $\phi_{c}$ and $\psi_{c}$ must change their sign. Thus, $\phi_{c}$ and $\psi_{c}$ must change their sign once and only once. And then by the step 1, we know that $\phi_{c}$ and $\psi_{c}$ cannot be both bounded.

We thus consider the following possible cases: 
(a) Both $\phi_{c}$ and $\psi_{c}$ change sign once and only once.

(b) $\phi_{c}(r) \geq 0$ and $\psi_{c}(r) \geq 0$ for $r \in[0, \infty)$.

(c) $\phi_{c}(r) \geq 0$ and $\psi_{c}(r)$ changes sign only once.

We need to exclude the last two cases.

CAse (b). Since

$$
\phi_{c}(r)=\left(A_{1}-B_{1} c\right) \log r+O(1) \text { at } \infty
$$

and

$$
\psi_{c}(r)=\left(-B_{2}+A_{2} c\right) \log r+O(1) \text { at } \infty,
$$

we have $A_{1}-B_{1} c \geq 0$ and $-B_{2}+A_{2} c \geq 0$ (but equality can hold only for one equation), which implies

$$
\frac{B_{2}}{A_{2}} \leq c \leq \frac{A_{1}}{B_{1}}
$$

i.e.,

$$
A_{1} A_{2}-B_{1} B_{2}>0 \text {. }
$$

But, we can prove that (see step 4 below)

$$
A_{1} A_{2}-B_{1} B_{2} \leq 0
$$

and it yields a contradiction. Hence this case is excluded.

CASE (c). Let $r_{i}$ be the last local maximum of $\phi_{c_{i}}(r)>0$. Hence,

$$
0 \leq-\Delta \phi_{c_{i}}\left(r_{i}\right)+e^{\left(u_{i}+v_{i}\right)\left(r_{i}\right)} \phi_{c_{i}}\left(r_{i}\right)=e^{v_{i}\left(r_{i}\right)}\left(1-e^{u_{i}\left(r_{i}\right)}\right) \psi_{c_{i}}\left(r_{i}\right),
$$

which implies $\psi_{c_{i}}\left(r_{i}\right)>0$. Thus,

$$
\psi_{c_{i}}(r)>0 \text { for } r \in\left[0, r_{i}\right] .
$$

If $r_{i} \rightarrow+\infty$, then we have $\psi_{c}(r) \geq 0$, a contradiction to the assumption of this case. Hence, $\left\{r_{i}\right\}$ are bounded, and then $\phi_{c}^{\prime}(r) \leq 0$ for large $r$. Thus, $\phi_{c}(r)$ is bounded.

For $\delta>0$, we denote

$$
\phi_{c+\delta}(r)=\left(\phi_{1}+c \phi_{2}+\delta \phi_{2}\right)(r) \text { and } \psi_{c+\delta}(r)=\left(\psi_{1}+c \psi_{2}+\delta \psi_{2}\right)(r) .
$$

ClaIm. If $\delta$ is small, then both $\phi_{c+\delta}$ and $\psi_{c+\delta}$ changes sign once and only once.

Suppose $\phi_{c+\delta}$ changes sign more than once. Since $\phi_{c+\delta}(r)<0$ for large $r$, we may assume that $\phi_{c+\delta}$ attains local positive maximum at $r_{2}(\delta)$. By the maximum principle,

$$
0 \leq-\Delta \phi_{c+\delta}\left(r_{2}(\delta)\right)+e^{(u+v)\left(r_{2}(\delta)\right)} \phi_{c+\delta}\left(r_{2}(\delta)\right)=e^{v\left(r_{2}(\delta)\right)}\left(1-e^{u\left(r_{2}(\delta)\right)}\right) \psi_{c+\delta}\left(r_{2}(\delta)\right),
$$


which implies $\psi_{c+\delta}\left(r_{2}(\delta)\right)>0$. Hence, $r_{2}(\delta) \leq C$ for some constant $C$ as $\delta$ is close to 0 .

Since $\phi_{c+\delta}$ changes sign more than once, there is a $r_{3}(\delta)<r_{2}(\delta)$ such that $\phi_{c+\delta}$ attains negative local minimum at $r_{3}(\delta)$.

Denote $r_{2}^{0}=\lim _{\delta \rightarrow 0} r_{2}(\delta)$ and $r_{3}^{0}=\lim _{\delta \rightarrow 0} r_{3}(\delta)$. By passing $\delta \rightarrow 0$, we have $\phi_{c}\left(r_{3}^{0}\right)=0$ and $\phi_{c}^{\prime}\left(r_{3}^{0}\right)=0$. As in step 2 , we have either $\psi_{c}\left(r_{3}^{0}\right)=0$ and $\psi_{c}^{\prime}\left(r_{3}^{0}\right)<0$, or $\psi_{c}\left(r_{3}^{0}\right)<0$. But it yields a contradiction to

$$
\psi_{c}\left(r_{2}^{0}\right)=\lim _{\delta \rightarrow 0} \psi_{c+\delta}\left(r_{2}(\delta)\right) \geq 0 .
$$

Similarly, we can prove $\psi_{c+\delta}$ changes sign once and only once. We conclude that

$$
\widehat{\alpha_{2}} \in S \text {. }
$$

Since $S$ is an open set, $\widehat{\alpha_{2}}+\varepsilon \in S$ for some $\varepsilon>0$ provided that $\widehat{\alpha_{2}}<\alpha_{2}^{*}$. Hence, $\widehat{\alpha_{2}}=\alpha_{2}^{*}$.

StEP 4. For any $\beta \in S$, then

$$
A_{1} A_{2}-B_{1} B_{2}<0 .
$$

Let $\phi_{c}\left(r ; \alpha_{1}^{*}, \beta\right)$ and $\psi_{c}\left(r ; \alpha_{1}^{*}, \beta\right)$ change sign once and only once. Then

$$
A_{1}-c B_{1} \leq 0
$$

and

$$
B_{2}-c A_{2} \leq 0
$$

Because the two inequalities cannot be equality simultaneously,

$$
A_{1} A_{2}-B_{1} B_{2}<0 \text {. }
$$

Hence, this theorem is proved.

\section{The Proof of Theorem 1.2}

Recall that

$$
\begin{aligned}
& \beta_{1}\left(\alpha_{1}, \alpha_{2}\right)=\int_{0}^{\infty} e^{v\left(r ; \alpha_{1}, \alpha_{2}\right)}\left(1-e^{u\left(r ; \alpha_{1}, \alpha_{2}\right)}\right) r d r \\
& \beta_{2}\left(\alpha_{1}, \alpha_{2}\right)=\int_{0}^{\infty} e^{u\left(r ; \alpha_{1}, \alpha_{2}\right)}\left(1-e^{v\left(r ; \alpha_{1}, \alpha_{2}\right)}\right) r d r .
\end{aligned}
$$

Hence,

$$
\frac{\partial \beta_{1}\left(\alpha_{1}, \alpha_{2}\right)}{\partial \alpha_{i}}=\int_{0}^{\infty}\left[\psi_{i}\left(r ; \alpha_{1}, \alpha_{2}\right) e^{v\left(r ; \alpha_{1}, \alpha_{2}\right)}\left(1-e^{u\left(r ; \alpha_{1}, \alpha_{2}\right)}\right)-\phi_{i}\left(r ; \alpha_{1}, \alpha_{2}\right) e^{(u+v)\left(r ; \alpha_{1}, \alpha_{2}\right)}\right] r d r
$$


and

$$
\frac{\partial \beta_{2}\left(\alpha_{1}, \alpha_{2}\right)}{\partial \alpha_{i}}=\int_{0}^{\infty}\left[\phi_{i}\left(r ; \alpha_{1}, \alpha_{2}\right) e^{u\left(r ; \alpha_{1}, \alpha_{2}\right)}\left(1-e^{v\left(r ; \alpha_{1}, \alpha_{2}\right)}\right)-\psi_{i}\left(r ; \alpha_{1}, \alpha_{2}\right) e^{(u+v)\left(r ; \alpha_{1}, \alpha_{2}\right)}\right] r d r
$$

$i=1,2$.

By Theorem 2.4, we have

$$
\operatorname{det}\left(\begin{array}{ll}
\frac{\partial \beta_{1}}{\partial \alpha_{1}} & \frac{\partial \beta_{1}}{\partial \alpha_{2}} \\
\frac{\partial \beta_{2}}{\partial \alpha_{1}} & \frac{\partial \beta_{2}}{\partial \alpha_{2}}
\end{array}\right)=\operatorname{det}\left(\begin{array}{cc}
A_{1} & -B_{1} \\
-B_{2} & A_{2}
\end{array}\right) \neq 0
$$

for $\left(\alpha_{1}, \alpha_{2}\right) \in \Omega$. Hence this theorem is proved.

\section{REFERENCES}

[1] L. Caffarelli and Y. Yang, Vortex condensation in the Chern-Simons-Higgs model: an existence theorem, Comm. Math. Phys. 168 (1995), 321-336.

[2] D. Chae and O. Imanuvilov, The existence of non-topological multivortex solutions in the relativistic self-dual Chern-Simons theory, Comm. Math. Phys. 215 (2000), 119-142.

[ 3 ] H. Chan, C. Fu AND C.-S. Lin, Non-topological multi-vortex solutions to the self-dual ChernSimons-Higgs equation, Comm. Math. Phys. 231 (2002), 189-221.

[4] J. Chern, Z. Chen AND C. Lin, Uniqueness of topological solutions and the structure of solutions for the Chern-Simons system with two Higgs particles, Comm. Math. Phys. 296 (2010), 323-351.

[5] K. ChOE, Multiple existence results for the self-dual Chern-Simons-Higgs vortex equation, Comm. Partial Differential Equations 34 (2009), 1465-1507.

[6] G. V. DunNe, Aspects of Chern-Simons theory, Aspects topologiques de la physique en basse dimension/Topological aspects of low dimensional systems, Les Houches, 1998, EDP Sci., Les Ulis, 1999, 177-263.

[7] A. Jaffe and C. Taubes, Vortices and monopoles: Structure of static gauge theories, Progress in physics 2, Birkhäuser Boston, Mass., 1980.

[ 8 ] C.-S. Lin, A. Ponce ANd Y. YANG, A system of elliptic equations arising in Chern-Simons field theory, Journal of Functional Analysis 247 (2007), 289-350.

[9] C.-S. Lin and J. Prajapat, Vortex condensates for relativistic abelian Chern-Simons model with two Higgs scalar fields and two gauge fields on a torus, Comm. Math. Phys. 288 (2009), 311-347.

[10] C.-S. LiN AND S. YAN, Bubbling solutions for relativistic abelian Chern-Simons model on a torus, Comm. Math. Phys. 297 (2010), 733-758.

[11] C.-S. LIN AND Y. YANG, Non-Abelian multiple vortices in supersymmetric field theory, Comm. Math. Phys. 304 (2011), 433-457.

[12] M. Nolasco and G. Tarantello, Vortex condensates for the SU(3) Chern-Simons theory, Comm. Math. Phys. 213 (2000), 599-639.

[13] J. Spruck AND Y. YAng, The existence of nontopological solitons in the self-dual ChernSimons theory, Comm. Math. Phys. 149 (1992), 361-376.

[14] G. Tarantello, Multiple condensate solutions for the Chern-Simons-Higgs theory, J. Math. Phys. 37 (1996), 3769-3796. 
Hsin-Yuan Huang

Department of Applied Mathematics

NATIONAL SUN YAT-SEN UNIVERSITY

KAOSHIUNG 804

TAIWAN

E-mail: hyhuang@math.nsysu.edu.tw

Chang-Shou Lin

Taida Institute for Mathematical Sciences

Center for Advanced Study in Theoretical Science

National TaIWAN University

TAIPEI, 106

TAIWAN

E-mail: cslin@tims.ntu.edu.tw 